\title{
EVEN SETS OF FOUR NODES ON RATIONAL SURFACES
}

\author{
Alberto Calabri, Ciro Ciliberto and Margarida Mendes lopes
}

Abstract. We describe smooth rational projective algebraic surfaces $X$, over an algebraically closed field of characteristic different from 2 , having an even set of four disjoint $(-2)$-curves $N_{1}, \ldots, N_{4}$, i.e. such that $N_{1}+\cdots+N_{4}$ is divisible by 2 in $\operatorname{Pic}(X)$.

\section{Introduction}

Let $X$ be a smooth projective algebraic surface over an algebraically closed field $\mathbf{k}$ of characteristic $\neq 2$. A set of $\nu$ disjoint nodal (i.e $(-2)$-curves) curves $N_{1}, \ldots, N_{\nu}$ is called an even set if there exists $L \in \operatorname{Pic}(X)$ such that $2 L \equiv$ $N_{1}+\cdots+N_{\nu}$. Since $K_{X} L=0, L^{2}$ is even and therefore the number $\nu$ is divisible by four because $4 L^{2}=-2 \nu$.

Assume that $X$ is rational, and that $N_{1}, \ldots, N_{\nu}$ is an even set of disjoint nodal curves with $\nu \geq 8$. Let $\eta: X \rightarrow \Sigma$ be the map which contracts the curves $N_{i}, i=1, \ldots, \nu$, to nodes.

By Theorem 3.2 of [DMP], there exists a fibration $f: \Sigma \rightarrow \mathbb{P}^{1}$ such that the general fibre of $f$ is a smooth rational curve and having $\nu / 2$ double fibres each containing two nodes of $\Sigma$.

Equivalently there exists a fibration $g: X \rightarrow \mathbb{P}^{1}$ with general smooth rational fibres, having $\nu / 2$ fibres, each containing two nodal curves $N_{k}, N_{l}$ and of the form $N_{k}+2 \Gamma+N_{l}$, where $\Gamma$ is a curve such that $\Gamma^{2}=-1, K_{X} \Gamma=-1$. Since a rational fibration has no multiple fibres, by Zariski's lemma (see [BPV]), such a curve $\Gamma$ is necessarily 1-connected and as such there is a birational morphism contracting $\Gamma$ to a smooth point. Remark that such a fibre can be obtained from a minimal model of the fibration by first blowing up a point $x$, then blowing up the double point of the total transform of the ruling through $x$, and possibly further blow-ups, cf. Example 1 in [DMP].

Note also that any set of $4 m$ of these nodal curves contained in $2 m$ such fibres is necessarily even.

Received July 9, 2004.

2000 Mathematics Subject Classification. 14J26.

Key words and phrases: rational surface, node, nodal curve, even sets of nodes.

The present collaboration takes place in the framework of the european contract EAGER, no. HPRN-CT-2000-00099. The first two authors are members of G.N.S.A.G.A.-I.N.d.A.M. The third author is a member of the Center for Mathematical Analysis, Geometry and Dynamical Systems, IST and was partially supported by FCT(Portugal) through program POCTI/FEDER and Project POCTI/MAT/44068/2002. 
By the proof of Theorem 3.2 of [DMP], an even set of four disjoint nodal curves orthogonal to the fibres of a fibration $g: X \rightarrow \mathbb{P}^{1}$, with smooth rational fibres, is necessarily contained in two fibres of $g$ as above.

Again by Theorem 3.3 in [DMP], if a rational surface $Y$ with Picard number $\rho(Y)=10-K_{Y}^{2}$ contains $\alpha, \alpha \geq 3$, disjoint nodal curves, then $\alpha \leq \rho(Y)-2$. Furthermore, if $\alpha=\rho(Y)-2$, then $\alpha=2 \beta$ is even, and $Y$ is obtained from a relatively minimal ruled rational surface $\mathbb{F}_{e}:=\operatorname{Proj}\left(\mathcal{O}_{\mathbb{P}^{1}} \oplus \mathcal{O}_{\mathbb{P}^{1}}(e)\right), e \geq 0$, with the process described above, i.e. by blowing up:

- $\beta$ points $p_{1}, p_{2}, \ldots, p_{\beta}$ in distinct fibres $F_{1}, F_{2}, \ldots, F_{\beta}$ of the same ruling of $\mathbb{F}_{e}$;

- the point $q_{i}, i=1,2, \ldots, \beta$, which is the intersection of the strict transform of $F_{i}$ with the exceptional curve over $p_{i}$.

We keep the terminology introduced in [DMP] and we will call $Y$ as above the standard example of a rational surface with $\rho(Y)-2$ disjoint nodal curves. For other terminology see "Notation and conventions" below.

In this note we complete the above results by proving the following:

Theorem 1.1. Let $X$ be a smooth rational surface containing an even set of 4 disjoint nodal curves $N_{1}, \ldots, N_{4}$. Then there exists a fibration $g: X \rightarrow \mathbb{P}^{1}$ with smooth rational fibres, having 2 fibres, each containing two nodal curves $N_{k}, N_{l}$ and of the form $N_{k}+2 \Gamma+N_{l}$, where $\Gamma$ is a curve such that $\Gamma^{2}=-1, K_{X} \Gamma=-1$.

From Theorem 1.1 we obtain:

Corollary 1.2. If $X$ is a smooth rational surface such that $X$ contains an even set of four nodal curves, then there is a birational morphism $\pi: X \rightarrow Y$, where $Y$ is a standard example with $K_{Y}^{2}=4$ and the morphism $\pi$ maps the four nodal curves of $X$ to the four nodal curves of $Y$.

Proof. Let $N_{1}, \ldots, N_{4}$ be the nodal curves. By Theorem 1.1, $X$ has a fibration $f$ : $X \rightarrow \mathbb{P}^{1}$ in smooth rational curves having fibres $N_{i}+2 \Gamma_{1}+N_{j}$ and $N_{k}+2 \Gamma_{2}+N_{l}$, $\{i, j, k, l\}=\{1,2,3,4\}$, and it is clear that, by contracting the $(-1)$-curves in the other fibres and possibly some curves contained in $\Gamma_{m}, m=1,2$, we get to a standard example.

Corollary 1.3. Let $X$ be a weak Del Pezzo surface containing an even set of four nodal curves.

Then there exists a birational morphism $\pi: X \rightarrow Y$, where $K_{Y}^{2}=4$ and $Y$ is obtained from $\mathbb{F}_{a}$, with $a=0$, 1 , or 2 , by blowing up:

- two points $p_{1}, p_{2}$ in distinct fibres $F_{1}, F_{2}$ of the same ruling of $\mathbb{F}_{a}$;

- the point $q_{i}, i=1,2$, which is the intersection of the strict transform of $F_{i}$ with the exceptional curve of $p_{i}$.

Furthermore, in case $a=2$, none of the blown-up points lies on the (-2)-curve of $\mathbb{F}_{2}$. 
Proof. Consider the birational morphism $\pi: X \rightarrow Y$ of Corollary 1.2. Since $-K_{X}$ is nef, also $-K_{Y}$ has to be nef. In particular, there are no rational curves $C$ on $Y$ or $X$ such that $C^{2} \leq-3$. This implies the assertion.

Our interest in these results arose in the course of investigating surfaces of general type with an involution, cf. [CCM]. However it seems to us of independent interest.

The main ingredients used for the proof of Theorem 1.1, which is presented in section 4, are some facts on adjoint systems on rational surfaces, which are collected in section 2, and Lemma 3.1, which is proved in section 3.

Notation and conventions. We work over any algebraically closed field $\mathbf{k}$ of characteristic $\neq 2$.

All surfaces are projective algebraic varieties of dimension 2 over $\mathbf{k}$. We do not distinguish between line bundles and divisors on a smooth variety. Linear equivalence is denoted by $\equiv$ and numerical equivalence over $\mathbb{Q}$ by $\sim$. The intersection product of divisors (line bundles) $A$ and $B$ is denoted by $A B$. As usual, given a divisor $D$ on a surface, $|D|$ will be the complete linear system of the effective divisors $D^{\prime} \equiv D$.

By a curve on a smooth surface $X$ we mean an effective, non zero divisor on $X$. However a $(-1)$-curve (resp. (-2)-curve or nodal curve) is an irreducible smooth rational curve $C$ such that $C^{2}=-1$ (resp. $\left.C^{2}=-2\right)$. A $(-1)$-divisor on $X$ will be a divisor $D$ satisfying $D^{2}=-1$ and $K_{X} D=-1$.

The Kodaira dimension of a surface $X$ is, as usual, denoted by $\kappa(X)$.

A smooth surface $X$ is called a weak del Pezzo surface if $-K_{X}$ is big and nef. The remaining notation is standard in algebraic geometry.

\section{Some properties of rational surfaces}

In this section we list some properties of rational surfaces, which we will need later. The properties on adjoint systems listed below can be also phrased in terms of Mori's theorem on the cone (cf. $[R]$ ), but here, for the reader's convenience, we state and prove them in the form we will need.

Lemma 2.1. Let $X$ be a rational surface. Then:

(i) If $D$ is a (-1)-divisor, then either $|D| \neq \emptyset$ or $\left|K_{X}-D\right| \neq \emptyset$.

(ii) Assume $-K_{X}$ is nef and big. Then each effective $(-1)$-divisor $D$ either contains a (-1)-curve or $K_{X}^{2}=1$ and $D \in\left|-K_{X}+A\right|=\left|-K_{X}\right|+A$, where $A$ is an effective divisor such that $K_{X} A=0, A^{2}=-2$.

Proof. The first assertion is an immediate consequence of the Riemann-Roch theorem, because $\chi\left(\mathcal{O}_{X}\right)=1$.

For (ii), we note that, if $D$ is not irreducible, since $-K_{X}$ is nef and big, there is one component $\Delta$ of $D$ such that $-K_{X} \Delta=1$ and every other component $\theta$ satisfies $-K_{X} \theta=0$. By the index theorem (see, e.g., Corollary 2.4 in [Ba]) and the adjunction formula, we see that $\theta^{2}=-2$ for each such $\theta$ and either $\Delta^{2}<0$ and $\Delta$ is a $(-1)$-curve or $\Delta^{2}=K_{X}^{2}=1$ and $\Delta \sim-K_{X}$. In the last case, 
$\Delta \equiv-K_{X}$ because numerical equivalence coincides with linear equivalence on rational surfaces.

Lemma 2.2. Let $D$ be a nef curve on a regular surface $X$ such that $p_{a}(D) \geq 1$. If $K_{X}+D$ is not nef, then any irreducible curve $\theta$ satisfying $\theta\left(K_{X}+D\right)<0$ is $a(-1)$-curve $\theta$ such that $\theta D=0$.

Proof. Since $X$ is regular, we have that $h^{0}\left(X, K_{X}+D\right) \geq p_{a}(D) \geq 1$.

Assume $K_{X}+D$ not nef. Then there is an irreducible curve $\theta$ such that $\theta\left(K_{X}+D\right)<0$. The curve $\theta$ is a component of the fixed part of $\left|K_{X}+D\right|$, and so $\theta^{2}<0$. Since $D$ is nef, we have $K_{X} \theta<0$, i.e. $\theta$ is a $(-1)$-curve and $\theta D=0$.

Lemma 2.3. Let $D$ be a curve on a rational surface $X$ such that $p_{a}(D)=1$ and $D^{2} \geq 1$. If $K_{X}+D$ is nef, then $D \equiv-K_{X}$. In particular $K_{X}^{2} \geq 1$.

Proof. As in the previous lemma, $h^{0}\left(X, K_{X}+D\right) \neq 0$. Since $\left(K_{X}+D\right) D=0$, the index theorem and the hypothesis $K_{X}+D$ nef imply that $K_{X}+D \sim 0$, hence $K_{X}+D \equiv 0$, because $X$ is rational.

Proposition 2.4. Let $D$ be a nef and big 2-connected curve on a rational surface $X$ with $K_{X}^{2} \leq 0$. Assume that $p:=p_{a}(D) \geq 2$ and $K_{X} D<0$. If $K_{X}+D$ is nef, then the following possibilities can occur:

(i) $\left(K_{X}+D\right)^{2}=0$, and $K_{X}+D \equiv(p-1) G$, where $|G|$ is a pencil of rational curves without base points such that $G D=2$;

(ii) $\left(K_{X}+D\right)^{2}>0$, and the general curve $D_{1}$ in $\left|K_{X}+D\right|$ is irreducible satisfying $p_{a}\left(D_{1}\right)<p_{a}(D)$.

Proof. Since $X$ is regular, $h^{0}\left(X, K_{X}+D\right)=p \geq 2$.

Write $\left|K_{X}+D\right|=|M|+F$, where $|M|$ is the moving part and $F$ the fixed part of the linear system $\left|K_{X}+D\right|$. Since $D$ is 2-connected, $\omega_{D}$ has no base points (see, e.g., $[\mathrm{CFM}]$, Proposition A.7, or $[\mathrm{M}]$ ) and so the nef divisor $D$ satisfies $D F=0$. This implies that, if $F \neq 0$, every curve $\theta$ contained in $F$ is such that $\theta D=0$ and so, by the index theorem, $\theta^{2}<0$. In particular, if $F \neq 0$, then $M F>0$, because $K_{X}+D$ is nef. Note that $D M=D(M+F)=D\left(K_{X}+D\right)$ is even, hence the equality $\left(K_{X}+D\right) M=M^{2}+M F$ implies, by the adjunction formula, that $M F$ is even.

Suppose that the general curve $M$ in $|M|$ is reducible. Then $|M|$ is composed with a pencil $|G|, p \geq 3, M \equiv(p-1) G$ and $G D=2$. Note that $p \geq 3$ implies in particular that $D^{2} \geq 5$, because $K_{X} D<0$. Since $G D=2$, the index theorem implies that $G^{2}=0$. Note that $G K_{X}=G F-G D=G F-2$ and thus $G F$ is even. Now $((p-1) G)\left(K_{X}+D\right)=(p-1) G F$. Since

$$
((p-1) G)\left(K_{X}+D\right) \leq\left(K_{X}+D\right)^{2}=K_{X}^{2}+K_{X} D+2(p-1)<2(p-1),
$$

we conclude that $G F=0$. So $F=0$ and we are in case (i). 
Suppose now that the general curve $M$ in $|M|$ is irreducible. We note that $h^{0}(X, M)=p$ and thus $h^{0}\left(M, \mathcal{O}_{M}(M)\right)=p-1$, because $X$ is regular. Now note that

$$
K_{X} M=\left(K_{X}+D\right) M-M D=M^{2}+M F-2(p-1),
$$

hence $K_{X} M+M^{2}=2 M^{2}+M F-2(p-1)$. Since

$$
M^{2}+M F \leq\left(K_{X}+D\right)^{2}=K_{X}^{2}+K_{X} D+2(p-1)<2(p-1),
$$

one has $K_{X} M<0$ and therefore the series $\mathcal{O}_{M}(M)$ is non special. By the Riemann-Roch theorem we obtain then

$$
p-1=M^{2}-M^{2}-\frac{1}{2} M F+(p-1),
$$

hence $M F=0$ and thus $F=0$. If $M^{2}=0$, we have $p=2$ and we are in case (i), whereas, if $M^{2}>0$, we are in case (ii).

In this case, since $K_{X}^{2} \leq 0$ and $K_{X} D<0$, we have necessarily $p_{a}(M)<$ $p_{a}(D)$.

\section{Even sets of nodes and double covers}

Let $X$ be a smooth projective algebraic surface. Given an even set of disjoint nodal curves $N_{1}, \ldots, N_{\nu}$ on $X$, let $\pi: Y \rightarrow X$ be the double cover branched on $N_{1}, \ldots, N_{\nu}$, defined by $2 L \equiv N_{1}+\cdots+N_{\nu}$ (cf. pg. 42 in [BPV]) and let $\eta: X \rightarrow \Sigma$, as in the Introduction, be the map that contracts the curves $N_{i}$ to nodes. The inverse image on $Y$ of a curve $N_{i}$ is a $(-1)$-curve $\Delta_{i}$. Blowing these $(-1)$-curves down to points $p_{1}, \ldots, p_{\nu}$, we obtain a smooth surface $\bar{Y}$ and a double cover $\bar{\pi}: \bar{Y} \rightarrow \Sigma$ branched precisely over the singularities of $\Sigma$. Then we have the following commutative diagram:

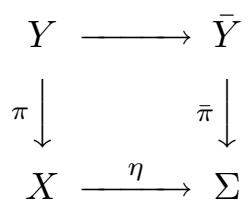

Note that $\Sigma$ has canonical singularities, so that $K_{\Sigma}$ is a Cartier divisor. Moreover $\bar{\pi}^{*}\left(K_{\Sigma}\right)=K_{\bar{Y}}$. Hence

$$
\begin{array}{ll}
\kappa(Y)=\kappa(\bar{Y})=\kappa(X), & K_{Y}^{2}=2 K_{X}^{2}-\nu, \\
K_{\bar{Y}}^{2}=2 K_{X}^{2}, & \chi\left(Y, \mathcal{O}_{Y}\right)=\chi\left(\bar{Y}, \mathcal{O}_{\bar{Y}}\right)=2-\frac{\nu}{4} .
\end{array}
$$

Finally we will need the following:

Lemma 3.1. Let $\bar{E}$ be a (-1)-curve of $\bar{Y}$ and let $E$ be the strict transform of $\bar{E}$ in $Y$. Then $E$ is a component of $\pi^{*}(C)$ where $C$ is an irreducible curve such that $K_{X} C=-1$, and such that, for each nodal curve $N_{i}$, either $C N_{i}=2$ or $C N_{i}=0$. 
Proof. Since $\bar{E}$ is a smooth curve, $E$ meets each of the $(-1)$-curves $\Delta_{i}$ transversally in at most one point. Note that $\sum \Delta_{i} \equiv \pi^{*}(L)$. Let $m$ be the number of curves $\Delta_{i}$ having non-empty intersection with $E$. Then $E^{2}=-1-m$ and $K_{Y} E=m-1$. Since $K_{Y} \equiv \pi^{*}\left(K_{X}+L\right)$, we conclude that $\pi^{*}\left(K_{X}\right) E=-1$. Since the map $H^{2}(X, \mathbb{Q}) \rightarrow H^{2}(Y, \mathbb{Q})$ induced by $\pi$ multiplies the intersection form by 2 , we conclude that the curve $E$ is not invariant under the involution $\iota$ of $Y$ associated to $\pi$. Then, if $C=\pi(E), \pi^{*}(C)=E+\iota(E)$ and $C$ is as stated.

\section{The proof of Theorem 1.1}

We use the notation of the statement of Theorem 1.1 and we denote again by $L$ the line bundle such that $N_{1}+\cdots+N_{4} \equiv 2 L$. The line bundle $L$ satisfies $L^{2}=-2, K_{X} L=0$ and $|L|=\emptyset$.

We will need the following:

Lemma 4.1. If there exists a (-1)-curve $E$ such that $E L=1$ and $E$ meets transversally exactly two of the nodal curves, say $N_{1}, N_{2}$, then $X$ is as in Theorem 1.1 .

Proof. Since $E L=1, E+L$ is a (-1)-divisor and therefore by Lemma 2.1, (i), either $|E+L| \neq \emptyset$ or $\left|K_{X}-(E+L)\right| \neq \emptyset$. The second possibility clearly does not occur, since, otherwise, $2 K_{X}$ would be effective. Therefore $|E+L| \neq \emptyset$. Since $N_{3} L=N_{4} L=-1$, we can write $E+L \equiv \Gamma+N_{3}+N_{4}$ where $\Gamma$ is an effective (-1)-divisor.

Note that $E(E+L)=0$ implies $E \Gamma=0$ and actually $E \cap \Gamma=\emptyset$. In fact otherwise $E$ would be a component of $\Gamma$, hence $E+L \equiv E+\Delta$, where $\Delta$ is an effective divisor, implying that $|L| \neq \emptyset$.

By the Riemann-Roch theorem, $h^{0}\left(X, 2 E+N_{1}+N_{2}\right) \geq 2$. Now, the relation $2 E+N_{1}+N_{2}+N_{3}+N_{4} \equiv 2(E+L) \equiv 2 \Gamma+2 N_{3}+2 N_{4}$ implies $2 E+N_{1}+N_{2} \equiv$ $2 \Gamma+N_{3}+N_{4}$. So $\left|2 E+N_{1}+N_{2}\right|$ is a pencil of rational curves without base points having fibres as in the statement.

Now we can give the:

Proof of Theorem 1.1. Since, by contracting (-1)-curves disjoint from $N_{1}, \ldots, N_{4}$, we still obtain a surface having an even set of 4 disjoint nodal curves, we will from now on make the following:

Assumption 4.2. There is no (-1)-curve on $X$ disjoint from the curves $N_{1}, \ldots, N_{4}$, i.e., for every (-1)-curve $E$, one has $E L \geq 1$.

We will argue by contradiction. So suppose that there is no fibration as in the statement. This implies that $K_{X}^{2}<4$, by Theorem 3.3 in [DMP]. Moreover, by Lemma 4.1 and Assumption 4.2, only the following two cases are possible:

(I) given a $(-1)$-curve $E$, one has $E L \geq 2$; thus by the index theorem $K_{X}^{2} \leq$ 1 , and $K_{X}^{2}=1$ if and only if $E L=2$ and $-K_{X} \equiv E+L$; or 
(II) given a (-1)-curve $E$, one has $E L=1$ and $E$ intersects exactly one of the nodal curves, say $E N_{1}=2$; thus by the index theorem $K_{X}^{2} \leq 1$ and $K_{X}^{2}=1$ if and only if $-K_{X} \equiv E+N_{1}$.

The surface $\bar{Y}$ as in Section 3 is not minimal, because $K_{\bar{Y}}^{2}<8$ and so there exists on $X$ an irreducible curve $C$ as in Lemma 3.1 meeting $m$ of the nodal curves, say $N_{1}, \ldots, N_{m}$ with $m \geq 0$, and satisfying $C L=m$. Since $C$ is irreducible and $K_{X} C=-1$, one has $C^{2} \geq-1$. Furthermore Assumption 4.2 means that, if $C^{2}=-1$, then necessarily $m>0$.

Set $D:=C+N_{1}+\cdots+N_{m}$. Remark that

$$
D N_{i}=0, i=1, \ldots, 4, \quad \text { and therefore } \quad D L=0 \text { and } D^{2}=C^{2}+2 m .
$$

The curve $D$ is nef, big and 2-connected. Since $K_{X} D=-1$, the index theorem yields $K_{X}^{2} \leq 1$, and

$$
K_{X}^{2}=1 \Longleftrightarrow-K_{X} \equiv D
$$

We start by considering this case.

4.1. The case $K_{X}^{2}=1$. Since $-K_{X} \equiv D, D^{2}=1$ and so either $D=C$ is irreducible or $C$ is a $(-1)$-curve intersecting only one of the nodal curves, say $N_{1}$, and $D=C+N_{1}$. We notice that in both cases $\left|-K_{X}\right|=|D|$ is a pencil without fixed components.

Since $K_{X}^{2}=1$, one has that $-K_{X}+L$ is a $(-1)$-divisor, and, by Lemma 2.1, (i), $\left|-K_{X}+L\right| \neq \emptyset$ because $-K_{X}\left(2 K_{X}-L\right)<0$. Since, for each $i=1, \ldots, 4$, $N_{i}\left(-K_{X}+L\right)=-1$, we can write $-K_{X}+L \equiv \Gamma+N_{1}+N_{2}+N_{3}+N_{4}$ where $\Gamma$ is an effective $(-1)$-divisor. Note that

$$
\Gamma+L \equiv-K_{X}
$$

4.1.1. Claim: $\Gamma$ is irreducible. Since $-K_{X}$ is nef and big, by Lemma 2.1, (ii), $\Gamma$ contains one $(-1)$-curve $\gamma$ which will satisfy one of the cases (I) or (II).

If $\gamma L \geq 2$, then, by case (I) and identity (1), we have $-K_{X} \equiv \gamma+L \equiv \Gamma+L$, which implies $\gamma=\Gamma$, i.e. the claim.

If $\gamma L=1$, then, again by (1), one has $\gamma \Gamma=0$. Since $\gamma$ is in case (II), there is a nodal curve $N_{i}$ such that $\gamma N_{i}=2$ and $\gamma+N_{i} \equiv-K_{X}$. Since $N_{i} \Gamma=$ $N_{i}\left(-K_{X}-L\right)=1$ by (1), then $N_{i} \gamma=2$ implies that $N_{i}$ is also a component of $\Gamma$. But, always by identity (1), one then has $-K_{X} \equiv \gamma+N_{i} \leq \Gamma \equiv-K_{X}-L$, which implies $-L \geq 0$, that is impossible.

4.1.2. Claim: every $(-1)$-curve $E \neq \Gamma$ satisfies $E L=1$, hence there is a nodal curve $N_{i}$ such that $E N_{i}=2$ and $-K_{X} \equiv E+N_{i}$. Suppose that $E$ is a (-1)curve such that $E L=2$. Then, by (I) and (1), one has $E+L \equiv-K_{X}$, hence $E=\Gamma$. The last assertions follow by case (II). 
4.1.3. Claim: there are $(-1)$-curves $E_{1}, E_{2}$, different from $\Gamma$, such that $E_{1} E_{2}=$ $1, E_{1} N_{1}=E_{2} N_{2}=2$ and $-K_{X} \equiv E_{1}+N_{1} \equiv E_{2}+N_{2}$. Since $-K_{X}$ moves in a pencil without fixed components and $-K_{X} N_{1}=0$, there is a curve $E_{1}+N_{1}$ in the pencil $\left|-K_{X}\right|$, where $E_{1}$ is an effective $(-1)$-divisor.

The curve $E_{1}$ is irreducible. Indeed, by Lemma 2.1, (ii), $E_{1}$ contains a (-1)curve $\theta$. Remark that $\theta \neq \Gamma$, otherwise by (1) we would have $\theta+L \equiv E_{1}+N_{1}$, which would imply $L>0$, a contradiction. Hence, by Claim 4.1.2, there exists one of the nodal curves $N_{i}$ such that $-K_{X} \equiv \theta+N_{i} \equiv E_{1}+N_{1}$. Since $\theta \leq E_{1}$ and $\left|-K_{X}\right|$ has no fixed components, this implies $\theta=E_{1}$.

The curve $E_{2}$ is found by applying the same reasoning to the fibre of the pencil $\left|-K_{X}\right|$ which contains $N_{2}$.

Since $-K_{X} \equiv E_{1}+N_{1} \equiv E_{2}+N_{2}$, for both curves $E_{1}, E_{2}$ we are in case (II), and not case (I). Therefore $E_{1} N_{2}=E_{2} N_{1}=0$, which implies that $E_{1} E_{2}=1$.

4.1.4. Claim: the linear system $\left|E_{1}+E_{2}\right|$ is a base point free pencil of rational curves, the curve $2 \Gamma+N_{3}+N_{4}$ sits in the pencil $\left|E_{1}+E_{2}\right|$, which has at least three reducible fibres. Notice that $-2 K_{X} \equiv E_{1}+N_{1}+E_{2}+N_{2} \equiv 2 \Gamma+N_{1}+N_{2}+N_{3}+N_{4}$, whence the first two assertions follow. For the last assertion, remark that $\rho(X)=$ 9, thus $\left|E_{1}+E_{2}\right|$ contains yet another reducible fibre.

Now we can conclude the proof for the case $K_{X}^{2}=1$.

A reducible fibre of $\left|E_{1}+E_{2}\right|$ contains at least one (-1)-curve $G$. So there is a $(-1)$-curve $G$ such that $G E_{1}=G E_{2}=G \Gamma=0$. Since $G \neq \Gamma$, one has $G L=1$ by Claim 4.1.1 and so $G$ is in case (II). On the other hand, $1=-K_{X} G=$ $G\left(E_{i}+N_{i}\right)=G N_{i}, i=1,2$, which is not possible in case (II).

4.2. The case $K_{X}^{2}<1$. We start with the following:

4.2.1. Claim: every (-1)-curve $E$ satisfies $E L \geq 2$, i.e. we are in case (I). Suppose otherwise, namely suppose there is a (-1)-curve $E$ for which case (II) holds, i.e. $E L=1, E N_{1}=2$ and $E N_{i}=0, i=2,3,4$. Hence the curve $A:=E+N_{1}$ is nef, $p_{a}(A)=1$ and $A L=0$. Since $\left(K_{X}+A\right)^{2}<0$, then $K_{X}+A$ is not nef and so, by Lemma 2.2, there exists a $(-1)$-curve $\theta$ such that $\theta A=0$. Then one has $(\theta+L) A=0$ and, therefore, $(\theta+L)^{2}<0$ by the index theorem. This implies $\theta L=1$, namely $\theta$ is as in case (II), i.e. there is a nodal curve, say $N_{2}$, such that $N_{2} \theta=2$. But then $\left(N_{2}+\theta\right)^{2}=1$ and $A\left(N_{2}+\theta\right)=0$, which contradicts the index theorem. This proves the claim.

Now we consider again the nef and big 2-connected curve $D:=C+N_{1}+\ldots+$ $N_{m}$, which satisfies $K_{X} D=-1$. In particular $p_{a}(D) \geq 1$.

4.2.2. Claim: $K_{X}+D$ is nef and moreover $D^{2} \geq 3, p_{a}(D) \geq 2$. Suppose that $K_{X}+D$ is not nef. By Lemma 2.2, there is a $(-1)$-curve $E$ such that $D E=0$. By Claim 4.2.1, one has $(E+L)^{2}>0$. Since $D L=0$, one also has $D(E+L)=0$. This gives a contradiction to the index theorem and so $K_{X}+D$ is nef. 
In particular $0 \leq\left(K_{X}+D\right)^{2}=K_{X}^{2}+2 K_{X} D+D^{2}$. Since $K_{X}^{2} \leq 0$ and $K_{X} D=-1$, we obtain $D^{2} \geq 2$. Since $D^{2}$ is odd by the adjunction formula, we have proved the last two assertions.

4.2.3. Claim: there is a positive dimensional linear system $|M|$ whose general curve $M$ is irreducible, smooth, rational and such that $M L=0$. We note first that $m K_{X}+D$ is orthogonal to $L$, for any $m \in \mathbb{N}$.

If $\left(K_{X}+D\right)^{2}=0$, by Proposition 2.4 one has $K_{X}+D \equiv(p-1) G$, where $|G|$ is a pencil of rational curves without base points and we have proven the claim. If $\left(K_{X}+D\right)^{2}>0$, again by Proposition 2.4 the general curve $D_{1} \in\left|K_{X}+D\right|$ is irreducible with $p_{a}\left(D_{1}\right)<p_{a}(D)$. If $p_{a}\left(D_{1}\right)=0$, again we proved the claim.

If $p_{a}\left(D_{1}\right)>0$ notice that, since $D_{1}$ is orthogonal to $L$ and $D_{1}^{2}>0$, we can show as in Claim 4.2.2 that $K_{X}+D_{1} \equiv 2 K_{X}+D$ is nef. So, by Lemma 2.3, $p_{a}\left(D_{1}\right) \geq 2$ and, as in the previous paragraph, by Proposition 2.4, either we find a linear system as in the claim or the general curve $D_{2} \in\left|K_{X}+D_{1}\right|$ is irreducible and satisfies $0<p_{a}\left(D_{2}\right)<p_{a}\left(D_{1}\right)<p_{a}(D)$.

It is clear that by iterating this procedure we eventually find a linear system as in the claim.

Now we can finish our proof of this case, and therefore of the theorem.

Consider the positive dimensional linear system $|M|$, whose existence is proved in Claim 4.2.3, and let $M$ be a general curve of $|M|$.

If $M^{2}=0$, then $|M|$ is a base-point-free pencil. Then by [DMP], as recalled in the introduction, the pencil $|M|$ is as in the statement of Theorem 1.1, contradicting our assumption.

If $M^{2}>0$, we also have a contradiction. Indeed, since $M$ is rational and smooth, $L$ is trivial on $M$ and thus, if $\pi: Y \rightarrow X$ is the double cover branched on $N_{1}+\cdots+N_{4}$, one has $\pi^{*}(M)=M_{1}+M_{2}$, where $M_{1} M_{2}=0$ and $M_{i}^{2}=M^{2}$. Since $M^{2}>0$, this contradicts the index theorem.

\section{References}

[Ba] L. Badescu, Algebraic surfaces, Universitext, Springer 2001.

[BPV] W. Barth, C. Peters, A. Van de Ven, Compact complex surfaces, Ergebnisse der Mathematik und ihrer Grenzgebiete, 3. Folge, Band 4, Springer 1984.

[CCM] A. Calabri, C. Ciliberto, M. Mendes Lopes, Numerical Godeaux surfaces with an involution, preprint.

[CFM] C. Ciliberto, P. Francia, M. Mendes Lopes, Remarks on the bicanonical map for surfaces of general type, Math. Z. 224 (1997), 137-166.

[DMP] I. Dolgachev, M. Mendes Lopes, R. Pardini, Rational surfaces with many nodes, Compositio Math. 132 (2002), 349-363.

[M] M. Mendes Lopes, Adjoint systems on surfaces, Boll. Un. Mat. Ital. A (7) 10 (1996), 169-179.

[R] M. Reid, Surfaces of small degree, Math. Ann. 275 (1986), 71-80. 
Dipartimento di Matematica, Università degli Studi di Bologna, Piazza di Porta San Donato, 5, I-40126 Bologna, Italy.

E-mail address: calabri@dm.unibo.it

Dipartimento di Matematica, Università degli Studi di Roma "Tor Vergata", Via Della Ricerca Scientifica, I-00133 Roma, Italy

E-mail address: cilibert@mat.uniroma2.it

Departamento de Matemática, Instituto Superior Técnico, Universidade Técnica de Lisboa, Av. Rovisco Pais, 1049-001 Lisboa, Portugal

E-mail address: mmlopes@math.ist.utl.pt 